ISAHP 1999, Kobe, Japan, August 12-14, 1999

\title{
Evaluation of Graduate Student Using an ANP Network Process
}

\author{
Han Wen Xiu and Wei Jin Yu \\ Institute of Systems Engineering, Management College \\ Tianjin University, Tianjin, China 300072 \\ Luo Yong Tai \\ Enterprise Management Department \\ Tianjin University of Finance \& Economics, Tianjin, China 300222 \\ LUOYONGTAI@YAHOO.COM
}

\begin{abstract}
More than three hundred graduated students are studying finance and economics course there at present in Tianjin University of Finance and Economics. Each of them studies a different course according to their private ability and level. How to rank their behavior and achievement becomes an urgent affair in graduate school. AHP Hierarchical Analysis may be the best solution to evaluate the graduate students' performance because it was a very useful implement before because we had used it to evaluate the graduate students' performance before. Now some graduate student points out that it is partial because some graduate students were part-time students, and some of them were doing more research than the others. And some others assist the professor do a lot of teaching jobs in the department. The AHP Hierarchical Analysis can not use the feedback and dependence when the dean makes the decision. So a new method to evaluate the graduate students' performance more impartial is introduced to the graduate school. It was ANP Network Process. With the ANP Network Process, the dean now can rank the graduate students fair because the judge can benefit its advantage of feedback and dependence variable. This paper presents this new method and narrates how to build the model with the criteria and sub-criteria that most graduate student concerned mostly. Supermatrix was applied to solve the problem that AHP Hierarchy Process can not deal with. Most graduate students in the university think the result that the ANP model generates are far more impartial than the previous AHP model's. The examples were given at the end of the paper to illustrate the usage of the ANP model we setup.
\end{abstract}

